\title{
INVERSE SPECTRAL BOUNDARY PROBLEM STURM LIOUVILLE TYPE WITH CONSTANT DELAY AND NON-ZERO INITIAL FUNCTION
}

\author{
MILENKO PIKULA ${ }^{1}$, DRAGANA NEDIĆ2* ${ }^{*}$, ISMET KALČO ${ }^{3}$, \\ LJILJANKA KVESIĆ 4 \\ ${ }^{1}$ Faculty of Philosophy, University of East Sarajevo \\ Bosnia and Herzegovina \\ ${ }^{2 *}$ Faculty of Transport and Traffic Engineering, University of East Sarajevo \\ Bosnia and Herzegovina \\ ${ }^{3}$ Faculty of Philosophy, University of Zenica \\ Bosnia and Herzegovina \\ ${ }^{4}$ Faculty of Science and Education, University of Mostar \\ Bosnia and Herzegovina \\ *Corresponding author. E-mail: dragana.nedic@sf.ues.rs.ba
}

\section{DOI: 10.20948/mathmontis-2021-51-2}

Summary. This paper is dedicated to solving of the direct and inverse spectral problem for Sturm Liouville type of operator with constant delay from $\frac{\pi}{2}$ to $\pi$, non-zero initial function and Robin's boundary conditions. It has been proved that two series of eigenvalues unambiguously define the following parameters: delay, coefficients of delay within boundary conditions, the potential on the segment from the point of delay to the right-hand side of the distance and the product of the starting function and potential from the left end of the distance to the delay point.

\section{INTRODUCTION}

Spectral theory represents a part of mathematical analysis which studies the spectrum, i.e. series of eigenvalues and vectors associated with linear operators defined on infinite dimensional functional spaces. Spectral problems can be divided into direct and inverse problems. Direct problems imply the constructions of characteristic functions, decomposition of functions from the domain of the operator according to the eigenfunctions of the operator, studying the asymptotics of their zeros as well as the asymptotics of eigenvalues of the operator. Inverse problems imply the construction of linear operator based on some of its known spectral characteristics. Fort he inverse problem of classic operator Sturm Liouville type, first results are obtained in papers [1], [2].

A current problematics that has been developing since the 1990s, (see [3],[4]) is exactly the one dealing with inverse spectral problems of Sturm Liouville type with removed argument.

The great number of published papers and significant results speak in favour of the fact that this field of mathemathics has developed intensly. Here are some of the results related to various types of delay. (see [3-24] )

This paper studies the direct and inverse spectral problem given with the following: 


$$
\begin{aligned}
& y^{\prime \prime}(x)+q(x) y(x-\tau)=\lambda y(x), \quad \lambda=z^{2}, \quad \tau \in\left[\frac{\pi}{2}, \pi\right) \\
& y(x-\tau)=\varphi(x-\tau), \quad x \in[0, \tau], \quad \varphi(0)=1 \\
& y^{\prime}(0)-h y(0)=0, \quad h \in R \\
& y^{\prime}(\pi)+H y(\pi)=0, \quad H \in R
\end{aligned}
$$

In papers [5-19] instead of the condition (2) we use the condition

$$
y(x-\tau) \equiv 0, x \in[0, \tau) .
$$

Let us have $q \in L_{2}[0, \pi], \varphi \in L_{2}[-\tau, 0]$.

The equation (1) with boundary condition (2) is equivalent to the integral equation

$$
y(x, z)=\cos x z+\frac{h}{z} \sin x z+\frac{1}{z} \int_{0}^{x} q\left(t_{1}\right) \sin z\left(x-t_{1}\right) y\left(t_{1}-\tau, z\right) d t_{1}
$$

Let us define the function

$$
\tilde{q}\left(t_{1}\right)=\left\{\begin{array}{cc}
q\left(t_{1}\right) \varphi\left(t_{1}-\tau\right), & t_{1} \in[0, \tau] \\
0, & t_{1} \in(\tau, \pi]
\end{array}\right.
$$

The solution of the equation (5) at distance $(0, \tau]$ is given with

$$
y(x, z)=\cos x z+\frac{h}{z} \sin x z+\frac{1}{z} \int_{0}^{x} \tilde{q}\left(t_{1}\right) \sin z\left(x-t_{1}\right) d t_{1}
$$

Next, we use the following functions

$$
\begin{aligned}
& a_{s c}(x, z)=\int_{\tau}^{x} q\left(t_{1}\right) \sin z\left(x-t_{1}\right) \cos z\left(t_{1}-\tau\right) d t_{1} \\
& a_{s^{2}}(x, z)=\int_{\tau}^{x} q\left(t_{1}\right) \sin z\left(x-t_{1}\right) \sin z\left(t_{1}-\tau\right) d t_{1} \\
& a_{s}^{(\widetilde{1})}(x, z)=\int_{0}^{\tau} \tilde{q}\left(t_{1}\right) \sin z\left(x-t_{1}\right) d t_{1} \\
& a_{s^{2}}^{(1, \widetilde{1})}(x, z)=\int_{\tau}^{x} q\left(t_{1}\right) \int_{0}^{t_{1}-\tau} \tilde{q}\left(t_{2}\right) \sin z\left(x-t_{1}\right) \sin z\left(t_{1}-\tau-t_{2}\right) d t_{2} d t_{1}
\end{aligned}
$$

At distance $(\tau, \pi]$ the solution of the equation (5) is given with

$$
\begin{gathered}
y(x, z)=\cos x z+\frac{h}{z} \sin x z+\frac{1}{z} a_{s}^{(\widetilde{1})}(x, z)+\frac{1}{z} a_{s c}(x, z)+ \\
+\frac{h}{z^{2}} a_{s^{2}}(x, z)+\frac{1}{z^{2}} a_{s^{2}}^{(1, \widetilde{1})}(x, z)
\end{gathered}
$$

\section{THE CONSTRUCTION OF CHARCTERISTIC FUNCTIONS OF $D^{2}$ OPERATOR}

The boundary problem given in (1-4) will be denoted shorter as $D^{2} y=\lambda y$. Let us construct a characteristic function of the operator $D^{2}$.

If we vary the condition (4) with $H$ to $H_{j}, j=1,2$ we obtain two characteristic equations $F_{j}(z)=0$.

Let us also introduce the following functions

$$
a_{c^{2}}(x, z)=\int_{\tau}^{x} q\left(t_{1}\right) \cos z\left(x-t_{1}\right) \cos z\left(t_{1}-\tau\right) d t_{1}
$$




$$
\begin{aligned}
& a_{c s}(x, z)=\int_{\tau}^{x} q\left(t_{1}\right) \cos z\left(x-t_{1}\right) \sin z\left(t_{1}-\tau\right) d t_{1} \\
& a_{c}^{(\widetilde{1})}(x, z)=\int_{0}^{\tau} \tilde{q}\left(t_{1}\right) \cos z\left(x-t_{1}\right) d t_{1} \\
& a_{c s}^{(1, \widetilde{1})}(x, z)=\int_{\tau}^{x} q\left(t_{1}\right) \cos z\left(x-t_{1}\right) \int_{0}^{t_{1}-\tau} \tilde{q}\left(t_{2}\right) \sin z\left(t_{1}-\tau-t_{2}\right) d t_{2} d t_{1}
\end{aligned}
$$

From (8) we obtain the following

$\frac{d}{d x} y(x, z)=-z \sin x z+h \cos x z+a_{c}^{(\widetilde{1})}(x, z)+a_{c^{2}}(x, z)+\frac{h}{z} a_{c s}(x, z)+\frac{1}{z} a_{c s}^{(1, \widetilde{1})}(x, z)$

Putting $x=\pi$ in (8) and (9), and then omit $\pi$ in the labels, for example $a_{c^{2}}(\pi, x)=a_{c^{2}}(x)$, based on the condition (4) the following is obtained

$$
\begin{aligned}
& F_{j}(z)=\left(-z+\frac{h H_{j}}{z}\right) \sin \pi z+\left(h+H_{j}\right) \cos \pi z+a_{c}^{(\widetilde{1})}(z)+a_{c^{2}}(z)+\frac{h H_{j}}{z^{2}}+ \\
& +a_{s^{2}}(z)+\frac{H_{j}}{z} a_{s}^{(\widetilde{1})}(z)+\frac{h}{z} a_{c s}(z)+\frac{H_{j}}{z} a_{s c}(z)+\frac{1}{z} a_{c s}^{(1, \widetilde{1})}(z)+\frac{H_{j}}{z^{2}} a_{s^{2}}^{(1, \widetilde{1})}(z)
\end{aligned}
$$

Let us transform the functions $F_{j}$ given in (10).

If we have

$$
\begin{aligned}
& \breve{q}(\theta)=\tilde{q}(2 \theta), \quad \hat{q}(\theta)=q\left(\theta+\frac{\tau}{2}\right), \quad I_{1}=\int_{\tau}^{\pi} q\left(t_{1}\right) d t_{1}=\int_{\frac{\tau}{2}}^{\pi-\frac{\tau}{2}} \hat{q}(\theta) d \theta \\
& \hat{a}(z)=\int_{\frac{\tau}{2}}^{\pi} \hat{q}(\theta) \cos (\pi-2 \theta) z d \theta, \quad \hat{b}(z)=\int_{\frac{\tau}{2}}^{\pi-\frac{\tau}{2}} \hat{q}(\theta) \sin (\pi-2 \theta) z d \theta
\end{aligned}
$$

Then the following relations are valid

$$
\begin{aligned}
& a_{c^{2}}(z)=\frac{1}{2} \hat{a}(z)+\frac{I_{1}}{2} \cos (\pi-\tau) z, \quad a_{s^{2}}(z)=\frac{1}{2} \hat{a}(z)-\frac{I_{1}}{2} \cos (\pi-\tau) z \\
& a_{c s}(z)=-\frac{1}{2} \hat{b}(z)+\frac{I_{1}}{2} \sin (\pi-\tau) z, \quad a_{s c}(z)=\frac{1}{2} \hat{b}(z)+\frac{I_{1}}{2} \sin (\pi-\tau) z
\end{aligned}
$$

Next, let us have

$$
\breve{a}(z)=\int_{0}^{\frac{\tau}{2}} \breve{q}(\theta) \cos (\pi-2 \theta) z d \theta, \quad \breve{b}(z)=\int_{0}^{\frac{\tau}{2}} \breve{q}(\theta) \sin (\pi-2 \theta) z d \theta
$$

Then we get

$$
a_{c}^{(\widetilde{1})}(z)=2 \breve{a}(z), \quad a_{s}^{(\widetilde{1})}(z)=2 \breve{b}(z)
$$

Then we transform the functions $a_{s^{2}}^{(1, \widetilde{1})}(z)$ and $a_{c s}^{(1, \widetilde{1})}(z)$.

By translating the product of trigonometric functions into sums, and then changing the order of integration, we obtain the following

$$
\begin{gathered}
a_{s^{2}}^{(1, \widetilde{1})}(z)= \\
=\int_{\frac{\tau}{2}}^{\frac{\pi}{2}}\left[\int_{\theta+\frac{\tau}{2}}^{2 \theta} q\left(t_{1}\right) \tilde{q}\left(2 t_{1}-2 \theta-\tau\right) d t_{1}-\tilde{q}(2 \theta-\tau) \int_{2 \theta}^{\pi} q\left(t_{1}\right) d t_{1}\right] \cos (\pi-2 \theta) z d \theta
\end{gathered}
$$




$$
+\int_{\frac{\pi}{2}}^{\pi-\frac{\tau}{2}} \int_{\theta+\frac{\tau}{2}}^{\pi} q\left(t_{1}\right) \tilde{q}\left(2 t_{1}-2 \theta-\tau\right) d t_{1} \cos (\pi-2 \theta) z d \theta
$$

Let us define the function $Q^{(1, \widetilde{1})}(\theta)$ with

$$
Q^{(1, \widetilde{1})}(\theta)=\left\{\begin{array}{c}
\int_{\theta+\frac{\tau}{2}}^{2 \theta} q\left(t_{1}\right) \tilde{q}\left(2 t_{1}-2 \theta-\tau\right) d t_{1}-\tilde{q}(2 \theta-\tau) \int_{2 \theta}^{\pi} q\left(t_{1}\right) d t_{1}, \quad \theta \in\left[\frac{\tau}{2}, \frac{\pi}{2}\right) \\
\int_{\theta+\frac{\tau}{2}}^{\pi} q\left(t_{1}\right) \tilde{q}\left(2 t_{1}-2 \theta-\tau\right) d t_{1}, \quad \theta \in\left[\frac{\pi}{2}, \pi-\frac{\tau}{2}\right]
\end{array}\right.
$$

and put

$$
\begin{aligned}
& a^{(1, \widetilde{1})}(z)=\int_{\frac{\tau}{2}}^{\pi-\frac{\tau}{2}} Q^{(1, \widetilde{1})}(\theta) \cos (\pi-2 \theta) z d \theta \\
& b^{(1, \widetilde{1})}(z)=\int_{\frac{\tau}{2}}^{\pi-\frac{\tau}{2}} Q^{(1, \widetilde{1})}(\theta) \sin (\pi-2 \theta) z d \theta
\end{aligned}
$$

Then the following relation is valid

$$
a_{s^{2}}^{(1, \widetilde{1})}(z)=a^{(1, \widetilde{1})}(z)
$$

Quite analogously, we obtain the following equation

$$
a_{c s}^{(1, \widetilde{1})}(z)=-b^{(1, \widetilde{1})}(z)
$$

Using the relations $\left(11_{l}\right), l=\overline{1,5}$, the functions in (10) obtain the following form

$$
\begin{gathered}
F_{j}(z)=\left(-z+\frac{h H_{j}}{z}\right) \sin \pi z+\left(h+H_{j}\right) \cos \pi z+2 \breve{a}(z)+\frac{2 H_{j}}{z} \breve{b}(z)+ \\
+\frac{I_{1}}{2}\left(1-\frac{h H_{j}}{z^{2}}\right) \cos (\pi-\tau) z+\frac{1}{2}\left(1+\frac{h H_{j}}{z^{2}}\right) \hat{a}(z)+\frac{I_{1}}{2 z}\left(h+H_{j}\right) \sin (\pi-\tau) z+ \\
+\frac{1}{2 z}\left(H_{j}-h\right) \hat{b}(z)-\frac{b^{(1, \tilde{1})}(z)}{z}+\frac{H_{j}}{z^{2}} a^{(1, \widetilde{1})}(z)
\end{gathered}
$$

Functions $F_{j}$ are entire functions of exponential type and unit increase rate at variable $z$.

\section{ASYMPTOTICS OF ZERO FUNCTION $F$}

Let us find the asymptotic of zero functions $F_{j}$.

From [3], the following is known to be valid

$$
z_{n j}=n+\frac{C_{1 j}(n)}{n}+\frac{C_{2 j}(n)}{n^{2}}+o\left(\frac{C_{2 j}(n)}{n^{2}}\right), \quad n \rightarrow \infty, j=1,2
$$

Therefore, we have

$$
\begin{aligned}
& \sin \pi z_{n j}=(-1)^{n}\left[\frac{\pi C_{1 j}(n)}{n}+\frac{\pi C_{2 j}(n)}{n^{2}}+o\left(\frac{C_{2 j}(n)}{n^{2}}\right)\right] \\
& -z_{n j} \sin \pi z_{n j}=(-1)^{n+1}\left[\pi C_{1 j}(n)+\frac{\pi C_{2 j}(n)}{n}+o\left(\frac{C_{2 j}(n)}{n}\right)\right]
\end{aligned}
$$




$$
\cos \pi z_{n j}=(-1)^{n}\left[1+O\left(\frac{\left(C_{1 j}(n)\right)^{2}}{n^{2}}\right)\right]
$$

We extend the function $\breve{q}$ from the segment $\left[0, \frac{\tau}{2}\right]$ to zero, by the space $[0, \pi]$, and if we have

$$
\check{a}_{2 n}=\frac{2}{\pi} \int_{0}^{\frac{\tau}{2}} \breve{q}(\theta) \cos 2 n \theta d \theta
$$

then the following is valid

$$
\check{a}\left(z_{n j}\right)=(-1)^{n} \frac{\pi}{2} \check{a}_{2 n}+o\left(\frac{C_{1 j}(n)}{n}\right)
$$

Similarly, if we have

$$
\check{b}_{2 n}=\frac{2}{\pi} \int_{0}^{\frac{\tau}{2}} \breve{q}(\theta) \sin 2 n \theta d \theta
$$

then we obtain

$$
\breve{b}\left(z_{n j}\right)=(-1)^{n+1} \frac{\pi}{2} \breve{b}_{2 n}+O\left(\frac{C_{1 j}(n)}{n}\right)
$$

Next, let us have

$$
\begin{aligned}
& \hat{a}_{2 n}=\frac{2}{\pi} \int_{\frac{\tau}{2}}^{\pi-\frac{\tau}{2}} \hat{q}(\theta) \cos 2 n \theta d \theta \\
& \hat{b}_{2 n}=\frac{2}{\pi} \int_{\frac{\tau}{2}}^{\pi-\frac{\tau}{2}} \hat{q}(\theta) \sin 2 n \theta d \theta
\end{aligned}
$$

then we obtain

$$
\hat{a}\left(z_{n j}\right)=(-1)^{n} \frac{\pi}{2} \hat{a}_{2 n}+o\left(\frac{1}{n}\right), \quad \hat{b}\left(z_{n j}\right)=(-1)^{n+1} \frac{\pi}{2} \hat{b}_{2 n}+o\left(\frac{C_{1 j}(n)}{n}\right)
$$

Next, the following is valid

$$
\begin{aligned}
& \cos (\pi-\tau) z_{n j}=(-1)^{n} \cos n \tau+(-1)^{n+1}(\pi-\tau) C_{1 j}(n) \frac{\sin n \tau}{n}+O\left(\frac{1}{n^{2}}\right) \\
& \sin (\pi-\tau) z_{n j}=(-1)^{n+1} \sin n \tau+O\left(\frac{1}{n}\right)
\end{aligned}
$$

Also, if we have

$$
b_{2 n}^{(1, \widetilde{1})}=\frac{2}{\pi} \int_{\frac{\tau}{2}}^{\pi-\frac{\tau}{2}} a^{(1, \widetilde{1})}(\theta) \sin 2 n \theta d \theta
$$

then we obtain

$$
b^{(1, \widetilde{1})}\left(z_{n j}\right)=(-1)^{n+1} \frac{\pi}{2} b_{2 n}^{(1, \widetilde{1})}+o\left(\frac{1}{n}\right)
$$

By using $\left(13_{l}\right), l=\overline{1,10}$ we come to the following estimates

$$
\begin{gathered}
F_{j}\left(z_{n j}\right)=(-1)^{n+1} \pi C_{1 j}(n)+(-1)^{n}\left(h+H_{j}\right)+(-1)^{n} \frac{I_{1}}{2} \cos n \tau+(-1)^{n} \pi \check{a}_{2 n}+ \\
+(-1)^{n} \frac{\pi}{4} \hat{a}_{2 n}+\frac{1}{n}\left[(-1)^{n+1} \pi C_{2 j}(n)+(-1)^{n+1} \pi \breve{b}_{2 n}+(-1)^{n+1} \frac{I_{1}(\pi-\tau)}{2} \cdot \frac{\sin n \tau}{n} C_{1 j}(n)\right.
\end{gathered}
$$




$$
\left.+(-1)^{n+1} \frac{I_{1}\left(h+H_{j}\right)}{2} \sin n \tau+(-1)^{n+1} \pi \frac{H_{j}-h}{2} \widehat{b}_{2 n}+(-1)^{n} \frac{\pi}{2} b_{2 n}^{(1, \widetilde{1})}\right]+o\left(\frac{1}{n^{2}}\right)=0
$$

Next,

$$
\begin{aligned}
C_{1 j}(n)= & \frac{h+H_{j}}{\pi}+\frac{I_{1}}{2 \pi} \cos n \tau+\check{a}_{2 n}+\frac{1}{4} \hat{a}_{2 n} ; \quad \check{a}_{2 n}+\frac{1}{4} \hat{a}_{2 n} \in l_{2} \\
C_{2 j}(n)= & \frac{I_{1}\left(h+H_{j}\right)}{2 \pi^{2}}(\tau-2 \pi) \sin n \tau-\frac{I_{1}^{2}(\pi-\tau)}{8 \pi^{2}} \sin 2 n \tau+\frac{1}{2 \pi} b_{2 n}^{(1, \widetilde{1})}- \\
& -\frac{H_{j}-h}{2} \widehat{b}_{2 n}-\check{b}_{2 n}-\frac{I_{1}(\pi-\tau)}{2 \pi} \sin n \tau\left(\check{a}_{2 n}+\frac{1}{4} \hat{a}_{2 n}\right)
\end{aligned}
$$

If we put

$$
\begin{gathered}
\zeta_{0 j}=\frac{h+H_{j}}{\pi}, \quad \zeta_{1}=\frac{I_{1}}{2 \pi}, \quad \zeta_{1 n}=\check{a}_{2 n}+\frac{1}{4} \hat{a}_{2 n}, \quad \eta_{1 j}=\frac{I_{1}\left(h+H_{j}\right)}{2 \pi^{2}}(\pi-2 \tau), \\
\eta_{2}=\frac{I_{1}^{2}(\pi-\tau)}{8 \pi^{2}}, \quad \eta_{2 n}=\frac{1}{2 \pi} b_{2 n}^{(1, \widetilde{1})}-\frac{H_{j}-h}{2} \widehat{b}_{2 n}-\check{b}_{2 n}-\frac{I_{1}(\pi-\tau)}{2 \pi} \zeta_{1 n}
\end{gathered}
$$

then we get

$z_{n j}=n+\frac{1}{n}\left(\zeta_{0 j}+\zeta_{1} \cos n \tau+\zeta_{1 n}\right)+\frac{1}{n^{2}}\left(\eta_{1 j} \sin n \tau+\eta_{2} \sin 2 n \tau+\eta_{2 n}\right)+o\left(\frac{\eta_{2 n}}{n^{2}}\right)$

In this way we have proved the result:

Theorem 3.1. Eigenvalues $\lambda_{n j}$ of boundary problems $\left(1,2,3,4_{j}\right), j=1,2$ have the asymptotic given with

$\lambda_{n j}=n^{2}+2 \zeta_{0 j}+2 \zeta_{1} \cos n \tau+2 \zeta_{1 n}+\frac{1}{n}\left(2 \eta_{1 j} \sin n \tau+2 \eta_{2} \sin 2 n \tau+2 \eta_{2 n}\right)+o\left(\frac{\eta_{2 n}}{n}\right)$

where we have $\eta_{2 n} \in l_{2}, \zeta_{1 n} \in l_{2}$.

\section{SETTING THE INVERSE TASK}

Let us have two series $\lambda_{n j}, n \in N_{0}, j=1,2$ of eigenvalues with asymptotics (15). If the series $\lambda_{n j}-n^{2}$ oscillate, that is if $I_{1} \neq 0$, it is well known that the values $\tau, I_{1}, h, H_{j}, j=1,2$ are uniquely determined.

Next, we study the possibility of determining functions $\breve{q}$ and $\hat{q}$.

Characteristic functions are constructed through Hadamard's products, so the following applies:

$$
F_{j}(z)=\pi \lambda_{0 j} \prod_{n=1}^{\infty} \frac{\lambda_{n j}}{n^{2}}\left(1-\frac{z^{2}}{\lambda_{0 j}}\right) \prod_{n=1}^{\infty}\left(1-\frac{z^{2}}{\lambda_{n j}}\right), j=1,2, \quad z \in C
$$

The process of constructing integral equations by functions $\check{q} \mathrm{i} \hat{q}$ is based on the following identities

$$
\begin{gathered}
\left(-z+\frac{h H_{j}}{Z}\right) \sin \pi z+\left(h+H_{j}\right) \cos \pi z+2 \breve{a}(z)+\frac{h H_{j}}{Z} \breve{b}(z)+ \\
+\frac{I_{1}}{2}\left(1-\frac{h H_{j}}{z^{2}}\right) \cos (\pi-\tau) z+\frac{1}{2}\left(1+\frac{h H_{j}}{z^{2}}\right) \hat{a}(z)+\frac{I_{1}}{2 z}\left(h+H_{j}\right) \sin (\pi-\tau) z+
\end{gathered}
$$




$$
+\frac{1}{2 z}\left(H_{j}-h\right) \hat{b}(z)-\frac{b^{(1, \widetilde{1})}(z)}{z}+\frac{H_{j}}{z^{2}} a^{(1, \widetilde{1})}(z)=F_{j}(z), \quad j=1,2
$$

Let us have

$$
\begin{aligned}
& A(z)=2 \frac{H_{2} F_{1}(z)-H_{1} F_{2}(z)}{H_{2}-H_{1}}+2 z \sin \pi z-2 h \cos \pi z \\
& B(z)=2 z \frac{F_{2}(z)-F_{1}(z)}{H_{2}-H_{1}}-2 h \sin \pi z-2 z \cos \pi z
\end{aligned}
$$

Identities (17) are equivalent with

$$
\begin{aligned}
& 4 \breve{a}(z)+\hat{a}(z)-h \frac{\hat{b}(z)}{z}-2 \frac{b^{(1, \widetilde{1})}(z)}{z}+I_{1} \cos (\pi-\tau) z+\frac{I_{1} h}{z} \sin (\pi-\tau) z=A(z) \\
& 4 \check{b}(z)+\hat{b}(z)-h \frac{\hat{a}(z)}{z}+2 \frac{a^{(1, \tilde{1})}(z)}{z}+I_{1} \sin (\pi-\tau) z-\frac{I_{1} h}{z} \cos (\pi-\tau) z=B(z)
\end{aligned}
$$

Then we carry out partial integration over quotients $\frac{\hat{b}(z)}{z}, \frac{b^{(1, \widetilde{1})}(z)}{z}, \frac{\hat{a}(z)}{z}$ and $\frac{a^{(1, \widetilde{1})}(z)}{z}$. First, we put

$$
\begin{aligned}
& a^{I^{1} \hat{q}}(z)=\int_{\frac{\tau}{2}}^{\pi-\frac{\tau}{2}}\left(\int_{\frac{\tau}{2}}^{\theta} \hat{q}\left(\theta_{1}\right) d \theta_{1}\right) \cos (\pi-2 \theta) z d \theta \\
& b^{I^{1} \hat{q}}(z)=\int_{\frac{\tau}{2}}^{\pi-\frac{\tau}{2}}\left(\int_{\frac{\tau}{2}}^{\theta} \hat{q}\left(\theta_{1}\right) d \theta_{1}\right) \sin (\pi-2 \theta) z d \theta \\
& a^{I^{1} Q^{(1, \widetilde{1})}}(z)=\int_{\frac{\tau}{2}}^{\pi-\frac{\tau}{2}}\left(\int_{\frac{\tau}{2}}^{\theta} Q^{(1, \widetilde{1})}\left(\theta_{1}\right) d \theta_{1}\right) \cos (\pi-2 \theta) z d \theta \\
& b^{I^{1} Q^{(1, \widetilde{1})}}(z)=\int_{\frac{\tau}{2}}^{\pi-\frac{\tau}{2}}\left(\int_{\frac{\tau}{2}}^{\theta} Q^{(1, \widetilde{1})}\left(\theta_{1}\right) d \theta_{1}\right) \sin (\pi-2 \theta) z d \theta
\end{aligned}
$$

The following relations are valid

$$
\begin{aligned}
& \frac{\hat{b}(z)}{z}=-I_{1} \frac{\sin (\pi-\tau) z}{z}+2 a^{I^{1} \hat{q}}(z) \\
& \frac{\hat{a}(z)}{z}=I_{1} \frac{\cos (\pi-\tau) z}{z}-2 b^{I^{1} \hat{q}}(z) \\
& \frac{b^{(1, \widetilde{1})}(z)}{z}=-\int_{\frac{\tau}{2}}^{\pi-\frac{\tau}{2}} Q^{(1, \widetilde{1})}(\theta) d \theta \frac{\sin (\pi-\tau) z}{z}+2 a^{I^{1} Q^{(1, \widetilde{1})}(z)} \\
& \frac{a^{(1, \widetilde{1})}(z)}{z}=\int_{\frac{\tau}{2}}^{\pi-\frac{\tau}{2}} Q^{(1, \widetilde{1})}(\theta) d \theta \frac{\cos (\pi-\tau) z}{z}+2 b^{I^{1} Q^{(1, \widetilde{1})}}(z)
\end{aligned}
$$

It is clearly seen that the following is valid $\int_{\frac{\tau}{2}}^{\pi-\frac{\tau}{2}} Q^{(1, \widetilde{1})}(\theta) d \theta=0$.

Thus, the identities $\left(18_{l}\right), l=1,2$ obtain the following form:

$$
\begin{aligned}
& 4 \check{a}(z)+\hat{a}(z)-2 h a^{I^{1} \hat{q}}(z)-2 a^{I^{1} Q^{(1, \widetilde{1})}}(z)=C(z) \\
& 4 \breve{b}(z)+\hat{b}(z)-2 h b^{I^{1} \hat{q}}(z)-2 b^{I^{1} Q^{(1, \widetilde{1})}}(z)=S(z)
\end{aligned}
$$

Here we have $C(z)=A(z)-2 h I_{1} \frac{\sin (\pi-\tau) z}{z}, S(z)=B(z)$ 
Let us have the set $E=\left\{m+i y, m \in N_{0}, y \in R\right\} \subset C$.

Since the set $E$ has the finite accumulation points, according to Vitali's theorem the system of identity $\left(19_{l}\right), l=1,2$ at $C$ is equivalent to the system of identity at $E$.

Therefore,

$$
\begin{gathered}
\check{a}(m+i y)=\int_{0}^{\frac{\tau}{2}} \breve{q}(\theta) \cos (m+i y)(\pi-2 \theta) d \theta= \\
=(-1)^{m}\left[\int_{0}^{\frac{\tau}{2}}(\breve{q}(\theta) \operatorname{ch}(\pi-2 \theta) y) \cos 2 m \theta d \theta+i \int_{0}^{\frac{\tau}{2}}(\breve{q}(\theta) \operatorname{sh}(\pi-2 \theta) y) \sin 2 m \theta d \theta\right] \\
\hat{a}(m+i y)=(-1)^{m}\left[\int_{\frac{\tau}{2}}^{\pi-\frac{\tau}{2}}(\hat{q}(\theta) \operatorname{ch}(\pi-2 \theta) y) \cos 2 m \theta d \theta+\right. \\
\left.+i \int_{\frac{\tau}{2}}^{\pi-\frac{\tau}{2}}(\hat{q}(\theta) \operatorname{sh}(\pi-2 \theta) y) \sin 2 m \theta d \theta\right] \\
\breve{b}(m+i y)=(-1)^{m}\left[-\int_{0}^{\frac{\tau}{2}}(\breve{q}(\theta) \operatorname{ch}(\pi-2 \theta) y) \sin 2 m \theta d \theta+\right. \\
\left.+i \int_{0}^{\frac{\tau}{2}}(\breve{q}(\theta) \operatorname{sh}(\pi-2 \theta) y) \cos 2 m \theta d \theta\right] \\
\hat{b}(m+i y)=(-1)^{m}\left[-\int_{\frac{\tau}{2}}^{\pi-\frac{\tau}{2}}(\hat{q}(\theta) \operatorname{ch}(\pi-2 \theta) y) \sin 2 m \theta d \theta+\right. \\
\left.+i \int_{\frac{\tau}{2}}^{\pi-\frac{\tau}{2}}(\hat{q}(\theta) \operatorname{sh}(\pi-2 \theta) y) \cos 2 m \theta d \theta\right]
\end{gathered}
$$

Besides,

$$
C(m+i y)=\alpha(m, y)+i \beta(m, y), \quad S(m+i y)=\gamma(m, y)+i \delta(m, y)
$$

Next, the following tags are used

$$
\begin{aligned}
& \check{a}(m+i y)=(-1)^{m}\left[\check{a}_{2 m}^{c h}(y)+i \check{b}_{2 m}^{s h}(y)\right], \quad \check{b}(m+i y)=(-1)^{m}\left[-\breve{b}_{2 m}^{c h}(y)+i \check{a}_{2 m}^{s h}(y)\right] \\
& \hat{a}(m+i y)=(-1)^{m}\left[\hat{a}_{2 m}^{c h}(y)+i \hat{b}_{2 m}^{s h}(y)\right], \quad \hat{b}(m+i y)=(-1)^{m}\left[-\hat{b}_{2 m}^{c h}(y)+i \hat{a}_{2 m}^{s h}(y)\right]
\end{aligned}
$$

The identities $\left(19_{l}\right), l=1,2$ are equivalent to the following system of identities

$$
\begin{aligned}
& 4 \check{a}_{2 m}^{c h}(y)+\hat{a}_{2 m}^{c h}(y)-2 h a_{2 m}^{I^{1} \hat{q} c h}(y)-2 a_{2 m}^{I^{1} Q^{(1, \widetilde{1})} c h}(y)=(-1)^{m} \alpha(m, y) \\
& 4 \breve{b}_{2 m}^{s h}(y)+\hat{b}_{2 m}^{s h}(y)-2 h b_{2 m}^{I^{1} \hat{q} s h}(y)-2 b_{2 m}^{I^{1} Q^{(1, \tilde{1})} s h}(y)=(-1)^{m} \beta(m, y) \\
& 4 \breve{b}_{2 m}^{c h}(y)+\hat{b}_{2 m}^{c h}(y)-2 h b_{2 m}^{I^{1} \hat{q} c h}(y)-2 h b_{2 m}^{I^{1} Q^{(1, \widetilde{1})} c h}(y)=(-1)^{m+1} \gamma(m, y) \\
& 4 \check{a}_{2 m}^{s h}(y)+\hat{a}_{2 m}^{s h}(y)-2 h a_{2 m}^{I^{1} \hat{q} s h}(y)-2 h a_{2 m}^{I^{I^{1} Q^{(1, \tilde{1})}} s h}(y)=(-1)^{m} \delta(m, y)
\end{aligned}
$$

According to the asymptotics (15) it is easy to conclude that the right-hand sides of $\left(20_{l}\right), l=\overline{1,4}$ are from the space $l_{2}$, in fact that Fourier's coefficients of some functions from $L_{2}[0, \tau]$ are known. From $\left(20_{1}\right)$ and $\left(20_{3}\right)$ we come to the following equation

$$
4 \breve{q}(\theta)+\hat{q}(\theta)-2 h \int_{\frac{\tau}{2}}^{\theta} \hat{q}\left(\theta_{1}\right) d \theta_{1}-2 \int_{\frac{\tau}{2}}^{\theta} Q^{(1, \widetilde{1})}\left(\theta_{1}\right) d \theta_{1}=\frac{f_{1}(\theta, y)}{\operatorname{ch}(\pi-2 \theta) y}
$$

Accordingly, from $\left(20_{4}\right)$ and $\left(20_{2}\right)$ at $y \neq 0$ we also have 


$$
4 \breve{q}(\theta)+\hat{q}(\theta)-2 h \int_{\frac{\tau}{2}}^{\theta} \hat{q}\left(\theta_{1}\right) d \theta_{1}-2 \int_{\frac{\tau}{2}}^{\theta} Q^{(1, \widetilde{1})}\left(\theta_{1}\right) d \theta_{1}=\frac{f_{2}(\theta, y)}{\operatorname{sh}(\pi-2 \theta) y}
$$

From $\left(21_{1}\right)$ and $\left(21_{2}\right)$ we obtain the following

$$
\frac{f_{1}(\theta, y)}{\operatorname{ch}(\pi-2 \theta) y}=\frac{f_{2}(\theta, y)}{\operatorname{sh}(\pi-2 \theta) y}=f(\theta), \theta \in[0, \pi],
$$

where equality is implied in terms of $L_{2}$.

In this way we have proved and important result:

Theorem 4.1. In order for functions $\breve{q}, \hat{q}$ to be parameters of operators $D_{j}^{2}(j=1,2)$ whose eigenvalues are given, it is necessary and sufficient for them to be the solutions of the equation

$$
4 \breve{q}(\theta)+\hat{q}(\theta)-2 h \int_{\frac{\tau}{2}}^{\theta} \hat{q}\left(\theta_{1}\right) d \theta_{1}-2 \int_{\frac{\tau}{2}}^{\theta} Q^{(1, \widetilde{1})}\left(\theta_{1}\right) d \theta_{1}=f(\theta)
$$

\section{SOLVING THE EQUATION (22)}

For $\theta \in\left[0, \frac{\tau}{2}\right]$ the equation (22) becomes the identity

$$
4 \check{q}(\theta)=f(\theta)
$$

that is

$$
q(x) \varphi(x-\tau)=\frac{1}{4} f\left(\frac{x}{2}\right), x \in[0, \tau]
$$

For $\theta \in\left(\frac{\tau}{2}, \pi-\frac{\tau}{2}\right]$ the equation (22) reduces to the following equation

$$
\hat{q}(\theta)=f(\theta)+\int_{\frac{\tau}{2}}^{\theta}\left[2 h \hat{q}\left(\theta_{1}\right)+2 Q^{(1, \widetilde{1})}\left(\theta_{1}\right)\right] d \theta_{1}
$$

or

$$
q\left(\theta+\frac{\tau}{2}\right)=f(\theta)+\int_{\frac{\tau}{2}}^{\theta}\left[2 h q\left(\theta_{1}+\frac{\tau}{2}\right)+2 Q^{(1, \widetilde{1})}\left(\theta_{1}\right)\right] d \theta_{1}
$$

i.e.

$$
q(x)=f\left(x-\frac{\tau}{2}\right)+\int_{\tau}^{x}\left[2 h q\left(x_{1}\right)+2 Q^{(1, \widetilde{1})}\left(x_{1}-\frac{\tau}{2}\right)\right] d x_{1}
$$

Since we have

$$
Q^{(1, \widetilde{1})}\left(x_{1}-\frac{\tau}{2}\right)=\left\{\begin{array}{c}
\int_{x_{1}}^{2 x_{1}-\tau} q\left(t_{1}\right) \tilde{q}\left(2 t_{1}-2 x_{1}\right) d t_{1}-\tilde{q}\left(2 x_{1}-2 \tau\right) \int_{2 x_{1}-\tau}^{\pi} q\left(t_{1}\right) d t_{1}, \quad x_{1} \in\left[\tau, \frac{\pi+\tau}{2}\right] \\
\int_{x_{1}}^{\pi} q\left(t_{1}\right) \tilde{q}\left(2 t_{1}-2 x_{1}\right) d t_{1}, \quad x_{1} \in\left(\frac{\pi+\tau}{2}, \pi\right]
\end{array}\right.
$$

the equation (24) is divided into two equations 


$$
\begin{gathered}
q(x)=f\left(x-\frac{\tau}{2}\right)+\int_{\tau}^{x}\left[2 h q\left(x_{1}\right)+\right. \\
\left.+\int_{x_{1}}^{2 x_{1}-\tau} q\left(t_{1}\right) \tilde{q}\left(2 t_{1}-2 x_{1}\right)-\tilde{q}\left(2 x_{1}-2 \tau\right) \int_{2 x_{1}-\tau}^{\pi} q\left(t_{1}\right) d t_{1}\right] d x_{1}, x \in\left(\tau, \frac{\pi+\tau}{2}\right] \\
q(x)=f\left(x-\frac{\tau}{2}\right)+\int_{\tau}^{x}\left[2 h q\left(x_{1}\right)+Q^{(1, \widetilde{1})}\left(x_{1}-\frac{\tau}{2}\right)\right] d x_{1}, x \in\left(\frac{\pi+\tau}{2}, \pi\right]
\end{gathered}
$$

or

$$
\begin{gathered}
q(x)=f\left(x-\frac{\tau}{2}\right)+\int_{\tau}^{\pi} 2 h q\left(x_{1}\right) d x_{1}+ \\
+\int_{\tau}^{\pi} Q^{(1, \widetilde{1})}\left(x_{1}-\frac{\tau}{2}\right) d x_{1}-2 h \int_{x}^{\pi} q\left(x_{1}\right) d x_{1}-\int_{x}^{\pi} Q^{(1, \widetilde{1})}\left(x_{1}-\frac{\tau}{2}\right) d x_{1}
\end{gathered}
$$

Since we have $\int_{\tau}^{\pi} q\left(x_{1}\right) d x_{1}=I_{1}$ and $\int_{\tau}^{\pi} Q^{(1, \widetilde{1})}\left(x_{1}-\frac{\tau}{2}\right) d x_{1}=0$ if we put

$$
\begin{aligned}
& f_{1}(x)=f\left(x-\frac{\tau}{2}\right)+2 h I_{1} \text { the equation (26) is equivalent to the equation } \\
& \qquad q(x)=f_{1}(x)+\int_{x}^{\pi}\left[2 h q\left(x_{1}\right)+\int_{x_{1}}^{\pi} q\left(t_{1}\right) \tilde{q}\left(2 t_{1}-2 x_{1}\right) d t_{1}\right] d x_{1}
\end{aligned}
$$

The equation (27) is Volterra's non homogenous linear integral equation of $q$ function, since $\tilde{q}$ is a known function.

Let $q_{1}(x)$ be the unique solution of the equation (27).

Let us now return to the equation (25).

We can write the following

$$
\begin{gathered}
q(x)=f\left(x-\frac{\tau}{2}\right)+ \\
\int_{\tau}^{\frac{\pi+\tau}{2}}\left[2 h q\left(x_{1}\right)+\int_{x_{1}}^{2 x_{1}-\tau} q\left(t_{1}\right) \tilde{q}\left(2 t_{1}-2 x_{1}\right) d t_{1}-\tilde{q}\left(2 x_{1}-2 \tau\right) \int_{2 x_{1}-\tau}^{\pi} q\left(t_{1}\right) d t_{1}\right] d x_{1}- \\
-\int_{x}^{\frac{\pi+\tau}{2}}\left[2 h q\left(x_{1}\right)+\int_{x_{1}}^{2 x_{1}-\tau} q\left(t_{1}\right) \tilde{q}\left(2 t_{1}-2 x_{1}\right) d t_{1}-\tilde{q}\left(2 x_{1}-2 \tau\right) \int_{2 x_{1}-\tau}^{\pi} q\left(t_{1}\right) d t_{1}\right] d x_{1}
\end{gathered}
$$

Since we have $\quad \int_{\tau}^{\frac{\pi+\tau}{2}} 2 h q\left(x_{1}\right) d x_{1}=2 h\left(I_{1}-\int_{\frac{\pi+\tau}{2}}^{\pi} q_{1}\left(x_{1}\right) d x_{1}\right)$

and

$$
\begin{gathered}
\int_{\tau}^{\frac{\pi+\tau}{2}}\left[\int_{x_{1}}^{2 x_{1}-\tau} q\left(t_{1}\right) \tilde{q}\left(2 t_{1}-2 x_{1}\right) d t_{1}-\tilde{q}\left(2 x_{1}-2 \tau\right) \int_{2 x_{1}-\tau}^{\pi} q\left(t_{1}\right) d t_{1}\right] d x_{1}= \\
=-\int_{\frac{\pi+\tau}{2}}^{\pi} \int_{x_{1}}^{\pi} q_{1}\left(t_{1}\right) \tilde{q}\left(2 t_{1}-2 x_{1}\right) d t_{1}
\end{gathered}
$$

therefore, we get

$$
q(x)=f\left(x-\frac{\tau}{2}\right)+
$$




$$
\begin{gathered}
+2 h\left(I_{1}-\int_{\frac{\pi+\tau}{2}}^{\pi} q_{1}\left(x_{1}\right) d x_{1}\right)-\int_{\frac{\pi+\tau}{2}}^{\pi} \int_{x_{1}}^{\pi} q_{1}\left(t_{1}\right) \tilde{q}\left(2 t_{1}-2 x_{1}\right) d t_{1} d x_{1}- \\
-\int_{x}^{\frac{\pi+\tau}{2}}\left[2 h q\left(x_{1}\right)+\int_{x_{1}}^{2 x_{1}-\tau} q\left(t_{1}\right) \tilde{q}\left(2 t_{1}-2 x_{1}\right) d t_{1}-\tilde{q}\left(2 x_{1}-2 \tau\right) \int_{2 x_{1}-\tau}^{\pi} q\left(t_{1}\right) d t_{1}\right] d x_{1}
\end{gathered}
$$

From $2 x_{1}-\tau \leq \frac{\pi+\tau}{2} \Leftrightarrow x_{1} \leq \frac{\pi+3 \tau}{4} \quad$ we can write

$$
\begin{gathered}
\int_{x_{1}}^{2 x_{1}-\tau} q\left(t_{1}\right) \tilde{q}\left(2 t_{1}-2 x_{1}\right) d t_{1}= \\
=\int_{x_{1}}^{\frac{\pi+3 \tau}{4}} q\left(t_{1}\right) \tilde{q}\left(2 t_{1}-2 x_{1}\right) d t_{1}+\int_{\frac{\pi+3 \tau}{4}}^{2 x_{1}-\tau} q_{1}\left(t_{1}\right) \tilde{q}\left(2 t_{1}-2 x_{1}\right) d t_{1}
\end{gathered}
$$

and

$$
\tilde{q}\left(2 x_{1}-2 \tau\right) \int_{2 x_{1}-\tau}^{\pi} q\left(t_{1}\right) d t_{1}=\tilde{q}\left(2 x_{1}-2 \tau\right) \int_{2 x_{1}-\tau}^{\frac{\pi+\tau}{2}} q\left(t_{1}\right) d t_{1}+\tilde{q}\left(2 x_{1}-2 \tau\right) \int_{\frac{\pi+\tau}{2}}^{\pi} q_{1}\left(t_{1}\right) d t_{1}
$$

Let us put

$$
\begin{gathered}
f_{2}(x)=f\left(x-\frac{\tau}{2}\right)+ \\
+2 h\left(I_{1}-\int_{\frac{\pi+\tau}{2}}^{\pi} q_{1}\left(x_{1}\right) d x_{1}\right)-\int_{\frac{\pi+\tau}{2}}^{\pi} \int_{x_{1}}^{\pi} q_{1}\left(t_{1}\right) \tilde{q}\left(2 t_{1}-2 x_{1}\right) d t_{1} d x_{1}- \\
-\int_{x}^{\frac{\pi+\tau}{2}}\left[\int_{\frac{\pi+\tau}{2}}^{2 x_{1}-\tau} q_{1}\left(t_{1}\right) \tilde{q}\left(2 t_{1}-2 x_{1}\right) d t_{1}+\tilde{q}\left(2 x_{1}-2 \tau\right) \int_{\frac{\pi+\tau}{2}}^{\pi} q_{1}\left(t_{1}\right) d t_{1}\right] d x_{1}
\end{gathered}
$$

The equation (25) is equivalent to the following equation

$$
\begin{gathered}
q(x)=f_{2}(x)- \\
-\int_{x}^{\frac{\pi+\tau}{2}}\left[2 h q\left(x_{1}\right)+\int_{x_{1}}^{\frac{\pi+\tau}{2}} q\left(t_{1}\right) \tilde{q}\left(2 t_{1}-2 x_{1}\right) d t_{1}-\tilde{q}\left(2 x_{1}-2 \tau\right) \int_{2 x_{1}-\tau}^{\frac{\pi+\tau}{2}} q\left(t_{1}\right) d t_{1}\right] d x_{1}
\end{gathered}
$$

Thus, we obtain a linear integral equation of Volterra's type.

If we write (28) in the operator form

$$
q=A q, q \in L_{2}\left[\tau, \frac{\pi+\tau}{2}\right]
$$

then for

$$
q^{(1)}, q^{(2)} \in L_{2}\left[\tau, \frac{\pi+\tau}{2}\right]
$$

we get

$$
\begin{aligned}
& \left|\left(A q^{(2)}-A q^{(1)}\right)(x)\right| \leq 3 M(\gamma-x)^{\frac{1}{2}}\left\|q^{(2)}-q^{(1)}\right\|, \\
& M=\max \{2|h|,\|\tilde{q}\|\}, \quad \gamma=\frac{\pi+\tau}{2}, \gamma-x<1
\end{aligned}
$$




$$
\begin{aligned}
& \left|\left(A^{2} q^{(2)}-A^{2} q^{(1)}\right)(x)\right| \leq(3 M)^{2} \frac{(\gamma-x)^{\frac{3}{2}}}{\frac{3}{2}}\left\|q^{(2)}-q^{(1)}\right\| \\
& \left|\left(A^{3} q^{(2)}-A^{3} q^{(1)}\right)(x)\right| \leq(3 M)^{3} \frac{(\gamma-x)^{\frac{5}{2}}}{\frac{3}{2} \cdot \frac{5}{2}}\left\|q^{(2)}-q^{(1)}\right\|
\end{aligned}
$$

Induction easily proves that the following is valid

$$
\left|\left(A^{m} q^{(2)}-A^{m} q^{(1)}\right)(x)\right| \leq(6 M)^{m} \frac{1}{(2 m+1) ! !}(\gamma-x)^{\frac{2 m+1}{2}}\left\|q^{(2)}-q^{(1)}\right\|
$$

Therefore,

$$
\left\|A^{m} q^{(2)}-A^{m} q^{(1)}\right\| \leq \frac{(6 M)^{m}}{(2 m+1) ! !}\left(\frac{\pi-\tau}{2}\right)^{m+2}\left\|q^{(2)}-q^{(1)}\right\|,
$$

which means that $A^{m}$ is the clamp operator, wherever $m$ is large enough. This means that there is a unique solution for $q_{2}(x)$ of the equation (28) at space $\left[\tau, \frac{\pi+\tau}{2}\right]$.

This is how we have proved the basic result:

Theorem 4.1. Using two sets of eigenvalues $\lambda_{n j}$ obtained by varying boundary conditions at the righthand end of the space $[0, \pi]$ under the condition $I_{1} \neq 0$, the parameters $\tau, h, H_{1}, H_{2}, q(x) \varphi(x-\tau), x \in[0, \tau]$ and $q(x), x \in[\tau, \pi]$ are uniquely determined.

\section{CONCLUSION}

The aim of the research but also the motivation of this paper is to contribute to the development of inverse spectral theory for operators with deviation. Some results concerning the inverse spectral problems for the classical operator are given in the literature, as well as the results related to different types of delay.

Here, the direct and inverse boundary value problem of the Sturm-Liouville type with constant delay and non - zero initial function is observed and studied. Using the condition, we solved the inverse problem and took one step in the development of this theory.

\section{REFERENCES}

[1] V. Ambarzumjan, "Uber eine Frage der Eigenwerttheorie “, Zeitshr.fr Physik, 53,690-695 (1929)

[2] G. Borg, "Eine Umkehrung der Sturm-Liouvillschen Eigenwertaufgabe", Acta Math.,78., 1-96 (1946)

[3] M. Pikula, "Determination of a Sturm-Liouville-type differential operator with delay argument from two spectra", Mat. Vesnik, 43 (3-4), 159-171 (1991)

[4] M. Pikula, „Ob opredelenii differenitcalnogo uravneniia s peremennym zapazdyvaniem“, Math. Montis, 6, 71-91 (1996)

[5] G. Freiling, V. A. Yurko, "Inverse problems for Sturm-Liouville differential operators with a constant delay", Appl. Math. Lett., 25, 1999-2004 (2012)

[6] V. Vladičić, M. Pikula, "An inverse problems for Sturm-Liouville- type differential equation with a constant delay", Sarajevo J. Math., 12 (24) 1, 83-88 (2016)

[7] V.A.Yurko, S.A. Buterin, M.Pikula, "Sturm-Liouville differential operators with deviating 
argument", Tamkang J. Math., 48 (1), 61-71 (2017)

[8] S. A. Buterin, M. Sat, "On the half inverse spectral problem for an integrodifferential operator", Inverse Probl. Sci. Eng., 25 (10), 1508-1518 (2017).

[9] S.A. Buterin, V.A. Yurko, "An inverse spectral problem for Sturm-Liouville operators with a large constant delay", AM P 9 (1), 17-27 (2019)

[10] V.A. Yurko "Recovering differential operators with retarded argument", Differential Equations, 4, 510-514 (2019)

[11] N.P. Bondarenko, V.A. Yurko, "An inverse problem for Sturm-Liouville differential operators with deviating argument", Appl. Math. Lett., 83, 140-144. (2018)

[12] V.A. Yurko, "An inverse spectral problem for second order differential operators with retarded argument", Results Math., 74 (2), (2019)

[13] M. Pikula, V. Vladičić, B. Vojvodić, "Inverse Spectral Problems for Sturm Liouville Operators with a Constant Delay Less than Half the Length of the Interval and Robin Boundary Conditions", Results Math. , 74 (1), 45 (2019)

[14] V.A. Yurko "Solution of Inverse Problems for Differential Operators with Delay", Transmutation Operators and Applications (Trends in Mathematics), Verlag: Springer International Publishing, (2020) doi: 10.1007/978-3-030-35914-0_21

[15] M. Pikula, D. Nedić, E. Čatrnja, "Partial inverse spectral problem for the Sturm-Liouville operator with delay", Sarajevo J. Math, 16 (29) (1), 41-54 (2020)

[16] B. Vojvodić, M. Pikula, V. Vladičić, F.A. Cetinkaya, "Inverse problems for differential operators with two delays larger than half the length of the interval and Dirichlet conditions", Turk. J. Math., 44 (3), 900-905 (2020)

[17] M. Pikula, E. Čatrnja, I. Kalčo, "Spectral problems for operators with deviating arguments", Hacet. J. Math. Stat., 47 (5), 1172-1183 (2018)

[18] V. Vladičić, N. Djurić, "Incomplete Inverse Problem for Sturm-Liouville Type Differential Equation with Constant Delay", Results Math., 74 (4), 161 (2019)

[19] B. Vojvodic, M. Pikula, "The boundary value problem for the operator Sturm-Liouville type with N constant delays and asymptotics of eigenvalues", Math. Montis., 35, 5-21 (2016)

[20] B. Vojvodić, N. Pavlović Komazec, "Inverse problems for Sturm-Liouvile operator with potential functions from $L_{2}[0, \pi]$ ", Math. Montis., 49, 28-38 (2020)

[21] M. Shahriari, "Inverse problem for Sturm-Liouville differential operators with two constant delays", Turk. J. Math. doi:10.3906/mat-1811-113, in press.

[22] J. B. Conway, "Function of One Complex Variable", Second Edition, Springer-Verlag, New York, (1995)

[23] H. Hochstadt, Integralequations, John Wiley and Sons Ltd, New York (1989)

[24] M. Sat, "Inverse problems for Sturm-Liouville operators with boundary conditions depending on a spectral parameter", Electr. J. Differ. Equ, 26, 1-7 (2017)

Received May 10, 2021 